\title{
Colonoscopy-Induced Splenic Injury: An Unusual Complication Warranting Urgent Attention
}

\author{
Anas K. Gremida ${ }^{\mathrm{a}, \mathrm{b}}$, Megan Q. Chan ${ }^{\mathrm{a}}$, Christine Y. Hachemª
}

\begin{abstract}
Colonoscopy is a safe, well-tolerated procedure routinely performed for diagnostic and therapeutic purposes of colorectal diseases. Although splenic injury is an uncommon complication, it is associated with a significant morbidity and mortality. Therefore, splenic injury should always be in the differential in any patient with abdominal pain or hemodynamic instability during or following colonoscopy. We present a case of splenic rupture during an elective colonoscopy.
\end{abstract}

Keywords: Colonoscopy; Splenic rupture; Splenic injury; Splenectomy

\section{Introduction}

Colonoscopy is a popular diagnostic and therapeutic procedure for colorectal diseases. According to the National Survey of Endoscopic Capacity (SECAP) conducted by Centers for Disease Control and Prevention (CDC) in 2002, approximately 14.2 million colonoscopies are performed annually in the United States and a capacity of 8.2 million more colonoscopies annually are predicted [1]. Although generally a safe procedure, colonoscopies are not without risks. The most common complications include intraluminal hemorrhage and colonic perforation with an incidence of $1-2 \%$ and $0.1-0.2 \%$, respectively $[1,2]$. Those two complications are typically associated with biopsies and polypectomies [3, 4]. Rarer complications include splenic rupture, pneumothorax, pneumoperitoneum, acute appendicitis, retroperitoneal abscess, colonic volvulus, hernia incarceration, diverticulitis, hepatic injury, pneumoscrotum, and mesenteric tears [3-11]. Colonoscopy-

\footnotetext{
Manuscript accepted for publication May 19, 2014

aDepartment of Gastroenterology, Saint Louis University Hospital, St Louis, MO 63110, USA

bCorresponding Author: Anas K. Gremida, Department of Internal Medicine, Saint Louis University Hospital, 3635 Vista Ave, St Louis, MO 63110, USA. Email:agremida@slu.edu
}

doi: http://dx.doi.org/10.14740/jmc1817w related splenic injury was first reported by Wherry and Zehner in 1974 [12]. As colonoscopies have become standard of care since their introduction in the 1960s, there has been an increasing rate of splenic rupture with more than $75 \%$ of cases reported after 2000 and $84 \%$ after 2005 [5]. While the incidence of splenic rupture is estimated at between 0.00005 and $0.017 \%$ [4], the true incidence is unknown. Furthermore, this complication may be under-reported due to reluctance to publicize morbidity or because cases are undetected [2]. With the increasing use of colonoscopy, physicians should be cognizant of this potentially fatal complication. In this report, we present a case of splenic injury during an elective colonoscopy.

\section{Case Report}

A 74-year-old male with recent diagnosis of squamous cell carcinoma of the tongue status post chemo-radiotherapy had a follow-up PET scan showing multiple intense tracer foci in the large bowel with interval increased metabolic activity. His past medical history was also significant for bradyarrythmia requiring a pacemaker, abdominal aortic aneurysm and emphysema. The patient had no history of prior colonoscopies or abdominal surgeries. The colonoscopy was performed by an experienced endoscopist to further evaluate the colonic lesions found on the PET scan. The procedure was done under sedation with propofol and the patient was placed in the left lateral position. The scope passed with some difficulty to the cecum. Per endoscopist, there was no excessive looping or abdominal pressure exerted. An $8 \mathrm{~mm}$ sessile polyp in the ascending colon was removed with hot snare and a larger $25 \mathrm{~mm}$ semi-pedunculated polyp in the ascending colon was removed piecemeal with hot snare and Roth Net. During the procedure, the patient became hypotensive and tachycardic. The procedure was immediately aborted, and the patient was resuscitated with IV fluids and placed in Trendelenburg position. The patient's systolic blood pressure recovered to $100 \mathrm{~mm} \mathrm{Hg}$. When the patient recovered from sedation, he was fully alert and oriented, complaining of diffuse abdominal pain that referred to the left shoulder. Physical examination was significant for diffuse rebound abdominal tenderness. Rectal exam was unremarkable. Shortly, the patient's blood pressure dropped again to $50 \mathrm{~s} / 40 \mathrm{~s}$, and he was transferred to the ICU and started on vasopressors. Initial laboratory workup showed an acute drop in hemoglobin 


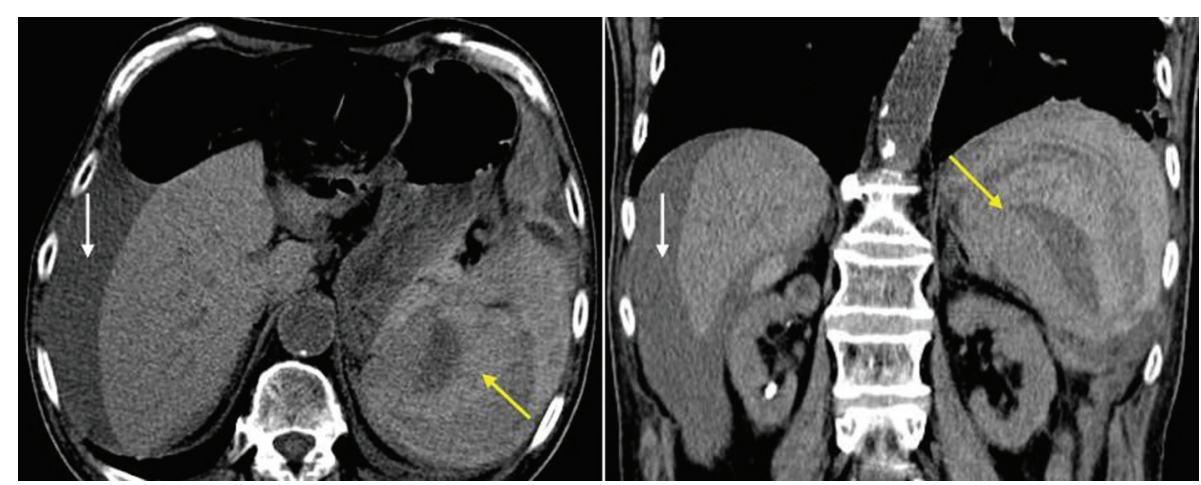

Figure 1. Non-contrast axial and coronal CT image of the abdomen shows a shattered spleen with a large amount of surrounding hemorrhage (yellow arrows) consistent with a grade 4-5 splenic injury. Hemoperitoneum extends to the perihepatic region (white arrows).

from 7.5 to $6.4 \mathrm{~g} / \mathrm{dL}$, a platelet count of $80,000 / \mathrm{mm}^{3}$ and a white blood cells count of $13,400 / \mathrm{mm}^{3}$. Two units of packed red blood cells were transfused. Abdominal X-rays showed no evidence of free air under the diaphragm. A non-contrast CT scan revealed a sub-capsular splenic hematoma and large volume hemo-peritoneum (Fig. 1). Hemoglobin repeat was $4.9 \mathrm{~g} /$ $\mathrm{dL}$. The patient was emergently taken for a laparotomy where surgeons found a ruptured spleen with an avulsed anterior capsule and $4 \mathrm{~L}$ of blood in the abdomen. No abdominal adhesions or colonic perforation were noted. Splenectomy and abdominal washout was performed. Surgical pathology showed a lacerated spleen with no underlying splenic pathology. The patient was extubated on postoperative day 2 and discharged 15 days after admission. Standard post-splenectomy vaccination was given prior to discharge. At a 3-week follow-up clinic appointment, the patient was doing well.

\section{Discussion}

Colonoscopy is the leading medical procedure in procedureinduced splenic rupture, followed by ERCP, liver surgery, and hysterectomy [6]. Diagnosis of splenic injury is often challenging because symptoms can be attributed to more common issues like colonic distension from retained gas, analgesia, perforation, or postpolypectomy coagulation syndrome [4]. Majority of patients present within 24 hours with the most common complaint of abdominal pain that may radiate to the left shoulder, referred to as "Kehr's sign", as in our reported case [13]. However there have also been reports of delayed presentation up to 10 - 13 days following colonoscopy $[7,8]$. Dense adhesions, which may increase the risk of splenic injury, may also delay the presentation by limiting the extent of the splenic hematoma and preventing peritoneal irritation. For unclear reasons, splenic injury occurs more in females. In a review of literature $72-76 \%$ of colonoscopy-related splenic injury occurred more in females who had similar numbers of colonoscopies compared to males with no extra risk factors $[11,13,14]$.

To date, the exact mechanisms of colonoscopy-induced splenic injury have not yet been fully elucidated. However, three main mechanisms have been postulated. The first is excessive traction on the splenocolic ligament secondary to certain endoscopic maneuvers causing torsional or tensile strain $[4-7,15-17]$. The second is traction on preexisting splenocolic adhesions from previous abdominal surgeries or inflammation $[4,6]$. The third postulated mechanism is direct splenic trauma as the endoscope traverses the splenic flexure, secondary to transcolic pressure, looping, or external pressure used to straighten the scope $[4,6,15]$. There is no consensus as to risk factors that predispose to splenic injury.

However, proposed risk factors can be divided into patientdependent risk factors and endoscopist-dependent risk factors (Table 1) $[4,5,7,14,15,17-22]$. Patient-dependent factors include previous intra-abdominal surgery, anticoagulation, inflammatory bowel disease, pancreatitis, and splenomegaly. Endoscopist-dependent predisposing factors include maneuvers that increase torque of the scope at the splenic flexure, such as hooking the splenic flexure to straighten the descending colon, slide by advancement, alpha maneuver, and intubation of the ileum $[12,17-19,23]$. Other techniques that may cause splenic injury include polypectomy, biopsies, excessive looping, and applying external pressure to the left upper quadrant, which induces rupture through a direct blunt trauma or decreased mobility between the spleen and colon [14, 18-21]. Placing patients in supine position has also been identified as a risk factor by causing the spleen to fall posteriorly away from the splenic flexure, increasing traction on the spleen during colonoscopy. Placing the patient in the left lateral position is preferred as this allows both the spleen and the splenic flexure to fall to the same side, slackening the splenocolic ligament and any adhesions in between [12].

Although technically difficult colonoscopies are proposed as a risk factor, the majority of splenic injuries reported have occurred in apparently uncomplicated colonoscopies without biopsies or polypectomies $[4,13]$. Even in cases that involved polypectomies, there was not a consistent site of polypectomy that led to an increased risk of splenic injury [19].

As in our reported case, we would like to highlight the fact that using deep sedation with propofol during colonoscopy procedures can also lead to masking the symptoms that may trigger the endoscopists' attention to an injury. Overall, any of 
Table 1. Risk Factors of Colonoscopy-Induced Splenic Injury [4, 5, 7, 15, 17-24]

\begin{tabular}{ll}
\hline Patient-dependent risk factors & Endoscopist-dependent risk factors \\
\hline Adhesions & Excessive traction on the splenocolic ligament \\
Prior intra-abdominal surgeries & Direct injury to spleen \\
Inflammatory intra-abdominal processes & Certain endoscopic maneuvers such as \\
Inflammatory bowel disease & Hooking the splenic flexure \\
Pancreatitis & Slide by advancement \\
Diverticulitis & Alpha maneuver \\
Splenomegaly & Straightening the sigmoid loop \\
Infectious disease & Intubation of ileum \\
Anticoagulation & Excessive looping \\
Neoplasm & External pressure \\
Multiple colonoscopies & Polypectomy \\
& Biopsy \\
& Technically difficult colonoscopy \\
& Supine position \\
& Operator inexperience \\
\hline
\end{tabular}

the above risk factors can theoretically predispose patients to splenic injury; however, there is insufficient evidence to confirm any of mentioned risk factors, likely due to the limited number of cases reported thus far.

CT scan remains the most sensitive diagnostic tool in detecting splenic injury $[13,16]$. Contrast-enhanced CT increases the accuracy of diagnosis to nearly $98 \%$. Contrast can also help grade the splenic injury according to the American Association of Surgeons for Trauma (AAST) Splenic Injury Grading Scale and helps identify active extravasation and other rare complications, such as hepatic injury or mesenteric hematomas [9]. Other less sensitive diagnostic studies include plain radiographs, ultrasound, peritoneal lavage, and laparotomy $[13,19]$. Plain radiographs can show raised left hemidiaphragm or leftsided pleural effusion, which may indicate splenic injury [15]. Focused assessment with sonography for trauma (FAST) is gaining popularity with $63-96 \%$ sensitivity for detecting intraabdominal fluid in hemodynamically unstable patients [24].

With a 5-8\% overall mortality rate for colonoscopy-associated splenic injury, workup should not be delayed if splenic damage is suspected $[4,15]$. The three most common treatment options include splenectomy, splenic artery embolization, and medical management. The majority of patients undergo operative intervention with subsequent splenectomy [13, $15,19]$. Splenic artery embolization was first reported in 2002 and has been used in low to high grade splenic ruptures [13, 19]. Laparoscopic splenectomy after colonoscopy-induced splenic rupture was first employed in 2012 [8]. Conservative medical management which typically entails volume repletion, broad-spectrum antibiotics and vigilant hemodynamic monitoring can be considered in hemodynamically stable patients with small, closed subcapsular hematomas [13, 25]. Predictors of failed conservative management include AAST grade III or higher injury, large hemoperitoneum, splenic hilar injury, older age, pre-existing splenic pathology, and the need for blood transfusions within the first $24 \mathrm{~h}[4,5,11]$. Luebke et al proposed practice guidelines for operative intervention, which include systolic blood pressure below $90 \mathrm{~mm} \mathrm{Hg}$, tachycardia over 120 beats/min, lack of response to $1-2 \mathrm{~L}$ of crystalloid resuscitation or when hemoperitoneum is evident [5].

\section{Conclusion}

Although colonoscopy-induced splenic injury is rare, it remains a serious complication with a significant mortality rate. A high index of suspicion is essential for prompt diagnosis and treatment and should be on the differential in patients presenting with abdominal pain or hemodynamic instability with a recent colonoscopy. The true incidence of colonoscopy-induced splenic injury is unknown and exact risk factors and mechanisms of injury have yet to be fully elucidated. Both CT scan and FAST have been used to confirm the diagnosis with contrast-enhanced CT being the preferred diagnostic tool. Decisions regarding treatment options rest largely on clinical judgment, but hemodynamic instability is a strong indication for surgical intervention. As awareness of colonoscopy-induced splenic injury increases, more patients will be identified, leading to improved understanding of this complication and optimization of patient outcomes.

\section{Financial Disclosure}

All authors have no financial interests to disclose.

\section{References}

1. Seeff LC, Richards TB, Shapiro JA, Nadel MR, Mannin- 
en DL, Given LS, Dong FB, et al. How many endoscopies are performed for colorectal cancer screening? Results from CDC's survey of endoscopic capacity. Gastroenterology. 2004;127(6):1670-1677.

2. Chow MD, Shih RD. Acute colonoscopy-induced splenic rupture presenting to the emergency department. Case Rep Emerg Med. 2013;2013:436874.

3. Sarhan M, Ramcharan A, Ponnapalli S. Splenic injury after elective colonoscopy. JSLS. 2009;13(4):616-619.

4. Ha JF, Minchin D. Splenic injury in colonoscopy: a review. Int J Surg. 2009;7(5):424-427.

5. Luebke T, Baldus SE, Holscher AH, Monig SP. Splenic rupture: an unusual complication of colonoscopy: case report and review of the literature. Surg Laparosc Endosc Percutan Tech. 2006;16(5):351-354.

6. Zandona C, Turrina S, Pasin N, De Leo D. Medico-legal considerations in a case of splenic injury that occurred during colonoscopy. J Forensic Leg Med. 2012;19(4):229233.

7. Volchok J, Cohn M. Rare complications following colonoscopy: case reports of splenic rupture and appendicitis. JSLS. 2006;10(1):114-116.

8. Abunnaja S, Panait L, Palesty JA, Macaron S. Laparoscopic splenectomy for traumatic splenic injury after screening colonoscopy. Case Rep Gastroenterol. 2012;6(3):624-628.

9. Koslowsky B, Adar T, Mahamid M, Melamud B, Goldin E, Ozick L. The unexpected twist in the plot: incarcerated diaphragmatic hernia complicating colonoscopy. Endoscopy. 2013;45 (Suppl 2 UCTN):E11.

10. Norena I, Varon A, Dennis C, Dennis R. Hepatic injury following colonoscopy. Endoscopy. 2013;45 (Suppl 2 UCTN):E247.

11. Tang RS, Hunt GC. Recurrent pneumothoraces after diagnostic colonoscopy. Gastroenterology. 2012;142(5):e9e10.

12. Wherry DC, Zehner H, Jr. Colonoscopy-fiberoptic endoscopic approach to the colon and polypectomy. Med Ann Dist Columbia. 1974;43(4):189-192.

13. Singla S, Keller D, Thirunavukarasu P, Tamandl D, Gupta S, Gaughan J, Dempsey D. Splenic injury during colon- oscopy--a complication that warrants urgent attention. J Gastrointest Surg. 2012;16(6):1225-1234.

14. Lalor PF, Mann BD. Splenic rupture after colonoscopy. JSLS. 2007;11(1):151-156.

15. Skipworth JR, Raptis DA, Rawal JS, Olde Damink S, Shankar A, Malago M, Imber C. Splenic injury following colonoscopy--an underdiagnosed, but soon to increase, phenomenon? Ann R Coll Surg Engl. 2009;91(4):W6-11.

16. Saad A, Rex DK. Colonoscopy-induced splenic injury: report of 3 cases and literature review. Dig Dis Sci. 2008;53(4):892-898.

17. Cappellani A, Di Vita M, Zanghi A, Cavallaro A, Alfano G, Piccolo G, Lo Menzo E. Splenic rupture after colonoscopy: Report of a case and review of literature. World J Emerg Surg. 2008;3:8.

18. Taylor FC, Frankl HD, Riemer KD. Late presentation of splenic trauma after routine colonoscopy. Am J Gastroenterol. 1989;84(4):442-443.

19. DuCoin C, Acholonu E, Ukleja A, Cellini F, Court I, Dabage N, Szomstein S, et al. Splenic rupture after screening colonoscopy: case report and literature review. Surg Laparosc Endosc Percutan Tech. 2010;20(1):e31-33.

20. Bergamaschi R, Arnaud JP. Splenic rupture from colonoscopy. Surg Endosc. 1997;11(11):1133.

21. Tse CC, Chung KM, Hwang JS. Prevention of splenic injury during colonoscopy by positioning of the patient. Endoscopy. 1998;30(6):S74-75.

22. Castelli M. Splenic rupture: an unusual late complication of colonoscopy. CMAJ. 1986;134(8):916-917.

23. Corcillo A, Aellen S, Zingg T, Bize P, Demartines N, Denys A. Endovascular treatment of active splenic bleeding after colonoscopy: a systematic review of the literature. Cardiovasc Intervent Radiol. 2013;36(5):1270-1279.

24. Prowda JC, Trevisan SG, Lev-Toaff AS. Splenic injury after colonoscopy: conservative management using CT. AJR Am J Roentgenol. 2005;185(3):708-710.

25. Poletti PA, Wintermark M, Schnyder P, Becker CD. Traumatic injuries: role of imaging in the management of the polytrauma victim (conservative expectation). Eur Radiol. 2002;12(5):969-978. 